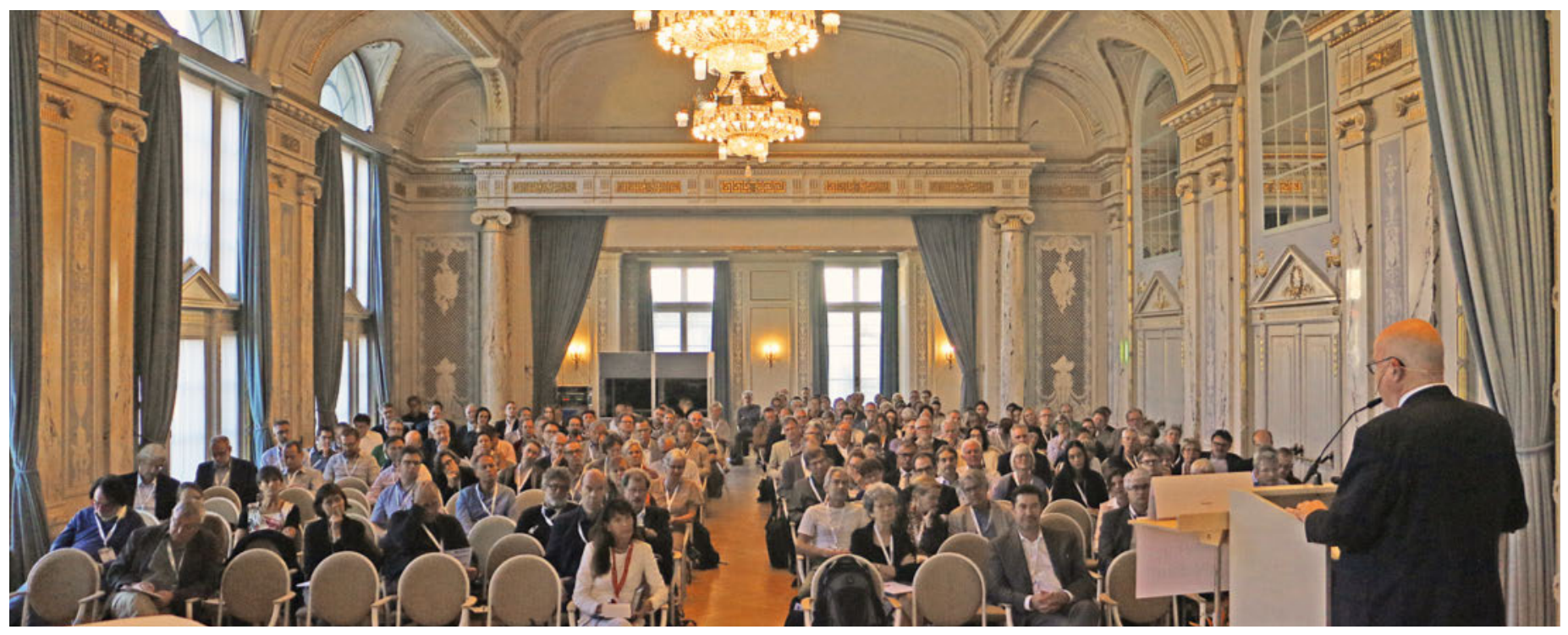

La troisième édition du symposium MedEd a une nouvelle fois suscité un vif intérêt de la part du public.

\title{
La formation postgraduée médicale: un patient qui a de l'avenir
}

\section{Bruno Kesseli}

Dr méd. et lic. phil., rédacteur en chef

La troisième édition du symposium MedEd de l'Institut suisse pour la formation médicale postgraduée et continue (ISFM) s'est tenue le 28 septembre à Berne. Les responsables de l'ISFM et son président Werner Bauer ont une nouvelle fois présenté un programme riche, informatif et passionnant.

Dans son discours d'ouverture, le président de l'ISFM a rappelé dans un clin d'œil au public que le terme de "symposium» est à l'origine synonyme de rencontre festive et de réjouissances. Si les organisateurs n'ont pas souhaité aller dans ce sens, ce symposium doit néanmoins être plus qu'une simple succession d'exposés et doit nourrir la réflexion et le débat afin de contribuer directement ou indirectement à l'amélioration de la formation postgraduée des médecins. Dans cet esprit, le regard vers l'étranger fait également partie du programme et permet de s'ouvrir à d'autres horizons.
Patients et médecins: des attentes en pleine évolution

Le symposium MedEd a ses traditions, comme le montrent les exposés de Pascal Strupler, qui s'est adressé aux participants lors des trois éditions. La question de la nécessité d'un tel symposium ne se pose désormais plus pour le directeur de l'Office fédéral de la santé publique, qui revient sur les deux premières éditions. Les débats ne se font jour que lorsqu'un thème est important et en pleine évolution. Des thèmes qui n'ont pas manqué lors des derniers sympo- 


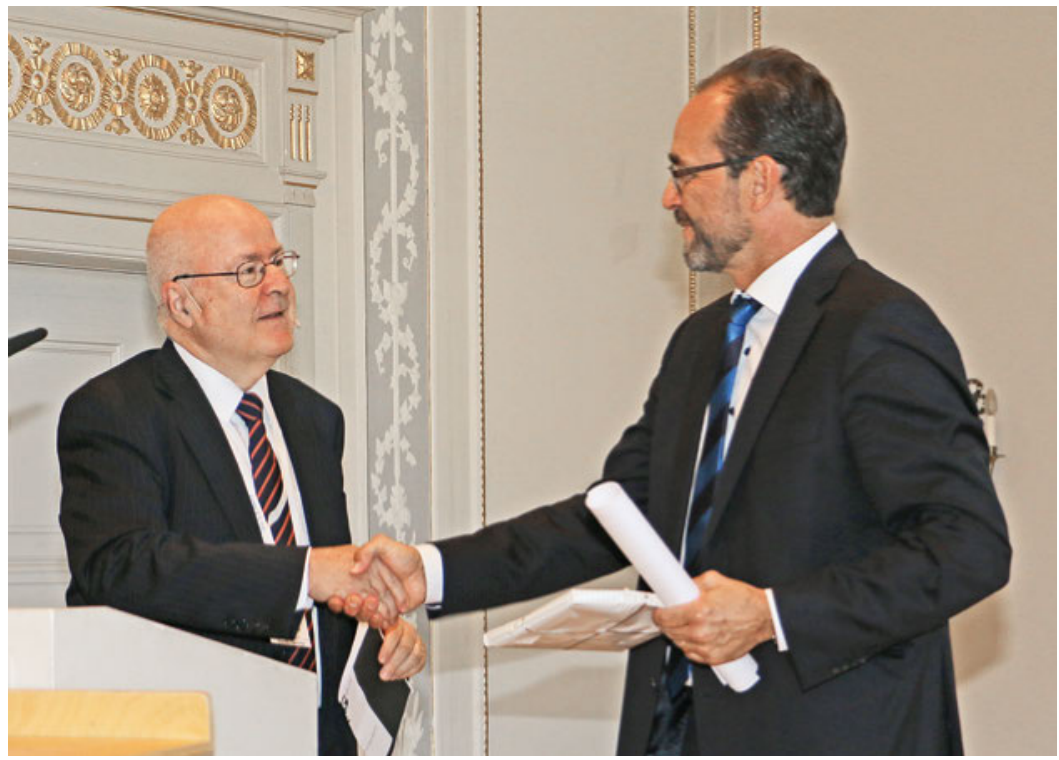

Werner Bauer (à g.) remet une petite attention à Pascal Strupler après sa présentation.

siums. Et le directeur de l'OFSP de rappeler à cet égard l'exposé un brin provocateur de Johann Steurer lors du 2 e symposium [1].

Soulignant par ailleurs l'importance du corps médical dans le système de santé, P. Strupler n'a pas manqué de préciser que celui-ci devait faire face aux défis de notre époque et être prêt au changement. Les médecins restent les piliers de notre système de santé. Et comme ils ne tombent pas du ciel ni ne peuvent être importés à volonté de l'étranger, «nous devons nous préoccuper de leur formation». Les attentes des patients comme des médecins sont en pleine évolution. Avec la stratégie Santé2020, le Conseil fédéral a tenté d'apporter des réponses efficaces aux défis d'aujourd'hui et de demain.

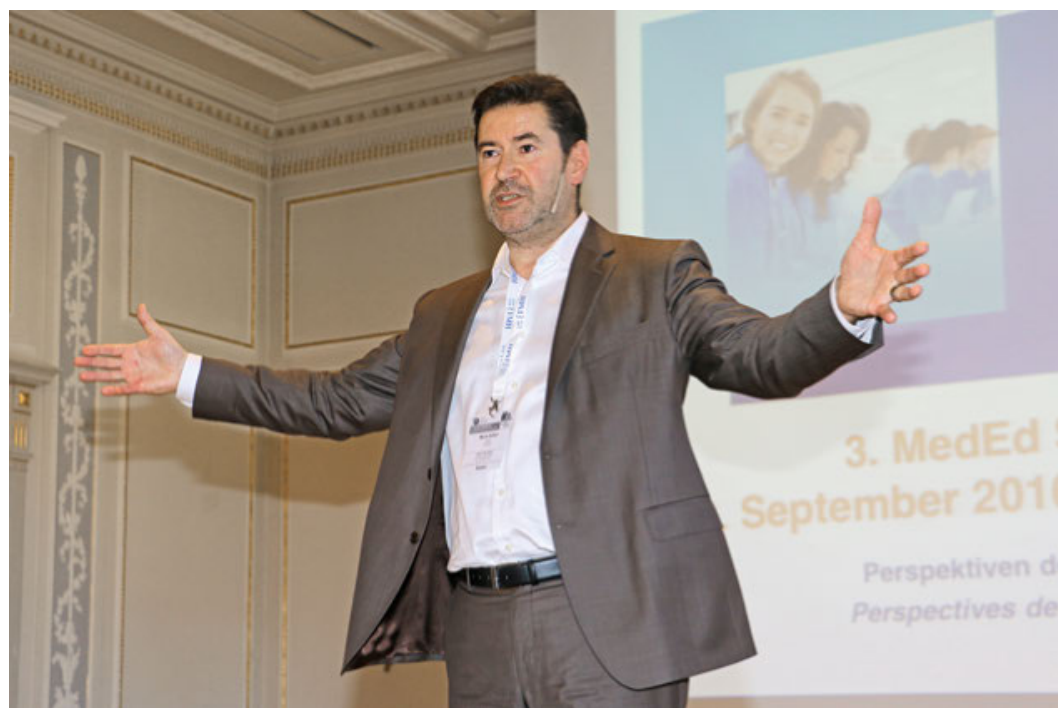

L'auto-organisation, un concept-clé: Marcel Altherr.
La question du pilotage, désormais plus souvent désignée sous le terme de coordination, reste actuelle à plusieurs niveaux et «interfaces»: de combien de spécialistes a-t-on besoin dans les différentes disciplines, comment atteindre les chiffres visés et comment amener les spécialistes dont on a besoin là où on en a besoin? Un autre sujet en discussion qui jouera un rôle important dans le système de santé de demain est celui de l'interprofessionnalité. P. Strupler a également laissé entrevoir que l'assouplissement voire la suppression de l'obligation de contracter restait à l'ordre du jour de différents acteurs de la santé, même s'il a conscience que le corps médical partage une position claire sur ce point.

\section{L'ISFM comme partie prenante}

Pour Werner Bauer aussi, tout n'est pas rose dans le système de santé et particulièrement dans le domaine de la formation médicale dont il est responsable. Un domaine qu'il a même volontiers désigné comme un "patient» dans le titre de son exposé [2]. Un patient mais avec un avenir, affirmant ainsi sa certitude qu'il existe des solutions aux questions actuelles. Pour W. Bauer, l'ISFM ne doit en aucun cas être une simple instance administrative mais a bien plus un rôle actif à jouer, explique-t-il à grand renfort d'images et de métaphores. A une époque où les méthodes et les structures sont en mouvement, la formation postgraduée doit elle aussi évoluer, continue-t-il. Les filets ne servent pas qu'à pêcher, et les clôtures, murets et autres silos ne doivent pas façonner ni bloquer les discussions sur la formation postgraduée. W. Bauer n'a pas le moindre doute que la formation postgraduée des futures générations de médecins est une obligation incontournable du corps médical. Pour cela, il faut toutefois disposer de temps et de moyens financiers, poursuit-il à l'intention des "partenaires» de la santé. Contrairement à la formulation des compétences à acquérir, une tâche exigeante dont «nous» - médecins - «devons nous préoccuper».

\section{La fièvre numérique s'étend}

Les maladies peuvent se propager à des institutions toutes entières, explique le mathématicien Marcel Altherr dans son exposé «La fièvre numérique». «Tout ce qui peut être numérisé sera numérisé tôt au tard», explique-t-il en s'inspirant de la célèbre citation de Dürrenmatt: «Tout ce qui est pensable sera pensé tôt au tard.» A son tour, il s'appuie sur des images fortes allant jusqu'à parler d'un «tsunami numérique», qui n'épargnera pas la formation postgraduée médicale. 


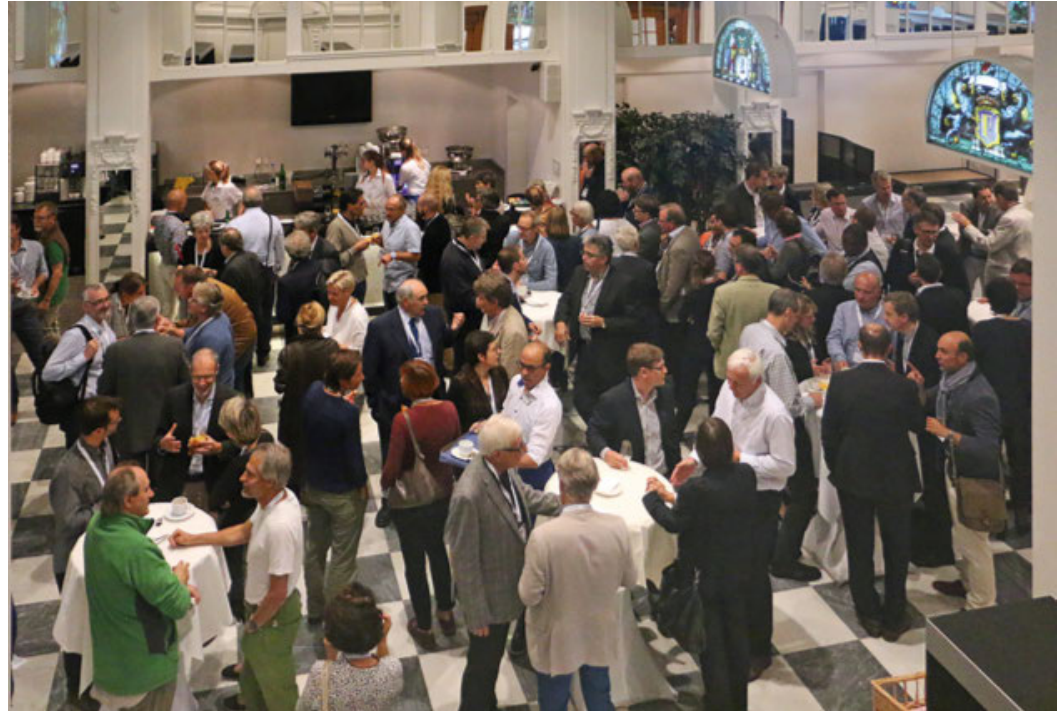

Une autre caractéristique du symposium MedEd: des débats engagés pendant les pauses.

S'il souligne que personne ne peut prédire à quoi ressemblera concrètement le nouveau paysage de la formation, il est clair pour lui que les organisations - y compris dans le domaine de la santé - auront à l'avenir besoin d'un nouveau "système d'exploitation" pour réussir. Car la théorie des organisations conçue au XIX siècle a désormais fait son temps même si elle continue de prédominer. "Auto-organisation» est pour lui le concept-clé pour le succès des organisations à l'avenir, avec le remplacement des hiérarchies classiques par des «cercles de responsabilité». Cette combinaison permet de faire émerger des «formes agiles» d'ores et déjà utilisées avec succès actuellement par différentes entreprises - aussi en Suisse. Et, fait intéressant, les

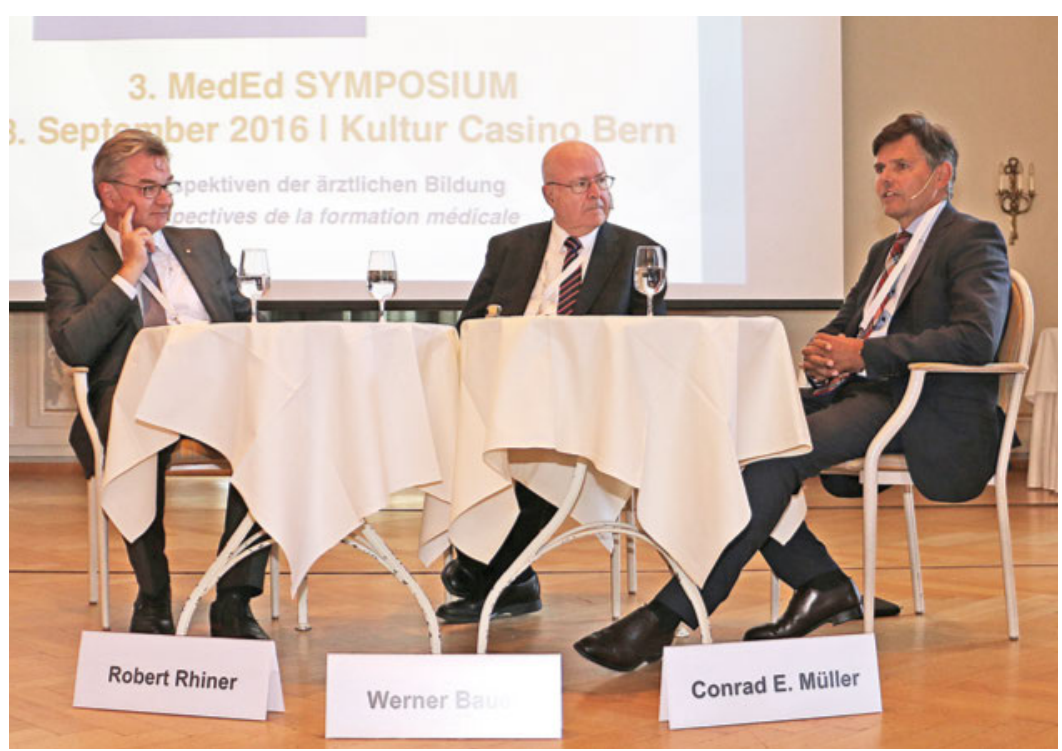

Entretien MedEd: Werner Bauer (au centre) s'entretient avec Robert Rhiner (à g.) et Conrad E. Müller. «bonnes vieilles valeurs», dont la confiance, continuent d'occuper une place importante dans ces modèles.

\section{Adhérer à la formation postgraduée malgré des conditions difficiles}

Lors de l'entretien MedEd, Werner Bauer s'est principalement intéressé à des questions d'actualité concrète en compagnie de deux médecins dirigeants d'hôpitaux: Robert Rhiner, directeur général de l'Hôpital cantonal d'Argovie, et Conrad E. Müller, directeur de la clinique Hirslanden Zurich. A plusieurs reprises, C. Müller a souligné que les cliniques Hirslanden comptaient s'engager dans la formation postgraduée. Sans rejeter la critique de Werner Bauer qui voit également les intérêts motivés par la médecine hautement spécialisée, il a toutefois précisé que ce n'était pas le facteur décisif. Après que quelques voix se sont élevées du public pour critiquer le nombre modeste de postes de formation en radiologie proposés par Hirslanden, C. Müller a expliqué que la mise en place de structures en ce sens prenait du temps mais que des fondations solides étaient désormais posées avec notamment un secrétariat à la formation. Robert Rhiner estime quant à lui que le niveau de formation dans son hôpital est élevé mais que le manque de temps et le caractère "contraignant" de l'enseignement pouvaient influer sur la qualité. Enfin, l'entretien a une fois de plus montré l'importance centrale de la question non encore résolue du financement de la formation postgraduée.

\section{Le point sur l'enseignement basé sur les compétences}

Comme bien d'autres domaines, la formation médicale connaît elle aussi des évolutions dont on ne sait pas précisément s'il s'agit de réels progrès ou d'un simple effet de mode. La tendance actuelle est à l'enseignement basé sur les compétences ou sur le résultat (Competency- ou Outcome-Based Education) dont font par exemple partie en Suisse aussi les «Entrustable Professional Activities» (EPA). Dans son exposé, le Canadien Geoff Norman porte un regard critique sur ces concepts et méthodes. Et personne ne reprochera à ce physicien nucléaire, psychologue et professeur émérite en épidémiologie clinique et biostatistique à la McMaster University, Canada, qu'il ne sait pas de quoi il parle. Une part importante de son travail est en effet consacrée à la formation médicale dont il analyse les instruments sous l'angle scientifique tout en participant activement à son développement. Auteur de nombreux ouvrages et articles sur la question de l'apprentissage et de la formation, il a su se faire un nom dans ce domaine. 


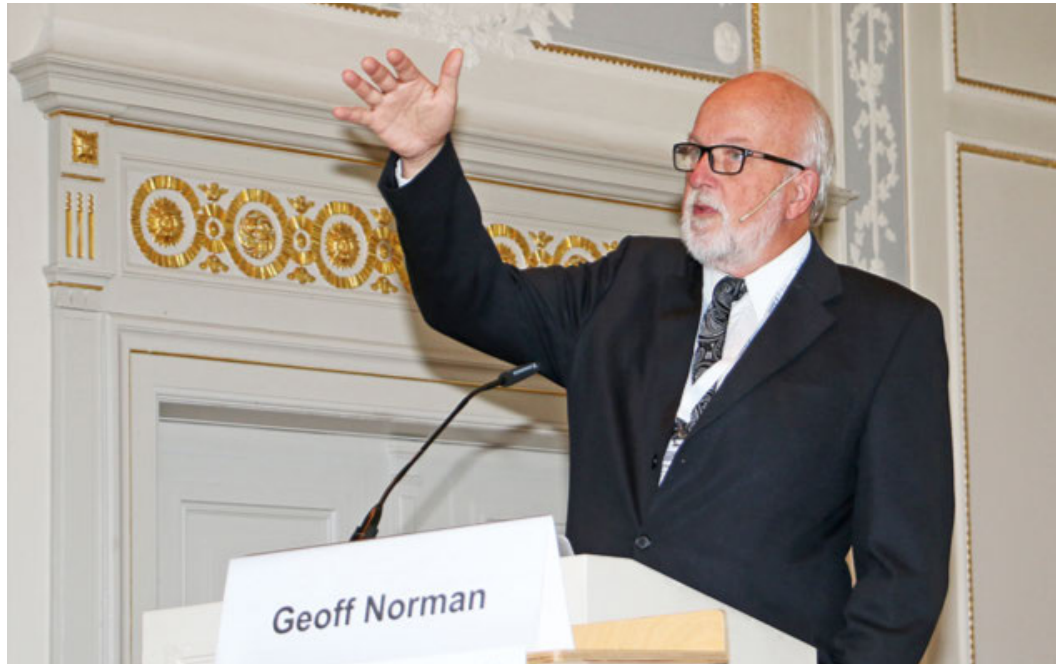

L'enseignement basé sur les compétences, une approche critique: Geoff Norman.

G. Norman a tout d'abord constaté que l'idée d'une éducation médicale basée sur les compétences suscitait une large adhésion. Mais en y regardant de plus près, il s'est rendu compte que «le diable se cachait dans les détails» et que les promesses de cette méthode n'étaient pas aussi simples à réaliser. L'idée selon laquelle une formation basée sur les compétences permet de réduire la durée de la formation pour atteindre un certain niveau de compétences, en d'autres termes, que cette forme d'éducation est plus efficace que les formes traditionnelles, n'y est sans doute pas étrangère. En outre, G. Norman a constaté que cette méthode ne permettait simplement pas de déterminer de manière fiable des niveaux de compétence tenant compte de l'ensemble des compétences acquises et d'établir une évaluation globale. Cela vaut d'ailleurs également pour les EPA.

Après ces explications, G. Norman a présenté sa solution qu'il nomme "Completed Clinical Evaluation Report Rating (CCER)». Cette approche associe micro-stratégie et macro-stratégie et consiste à évaluer différentes tâches spécifiques (niveau micro) à l'aide de listes de contrôle puis de les intégrer dans une évaluation globale (niveau macro). Lorsque ces évaluations sont réalisées régulièrement (quotidiennement, dans l'étude présentée), cette méthode permet de documenter de manière claire les progrès de la personne en formation avec un degré élevé de fiabilité. L'avenir dira si le CCER se diffusera et fera école également sous nos latitudes.

\section{Est-ce mieux sans temps et sans argent?}

L'après-midi a été consacrée à trois séminaires parallèles [2] sur les thèmes suivants:

- Et si le progrès technologique échappait aux experts de la formation?

- La formation postgraduée de demain: des cursus structurés en réseau?

- Diagnostic error mechanisms and prevention: an utopia?

Martin Krause s'est ensuite intéressé à une question dont la formulation se veut plutôt sarcastique: «La formation postgraduée réglementée sans temps ni argent: est-ce vraiment mieux?» Le récit très humoristique et bourré d'anecdotes du médecin-chef interniste de l'Hôpital de Münsterlingen a suscité de larges rires dans le public, signe de sa très certaine approbation. $M$ Krause a plaidé pour une médecine proche du patient, dans laquelle l'examen clinique et l'attention accordée au patient continuent à jouer un rôle essentiel. Pour lui, cela ne peut qu'avoir un impact positif sur la qualité de la formation postgraduée tout en répondant au désir des médecins-assistants, qui apprécient la proximité des patients. Pour lui, l'ISFM fournit un excellent

«Proche du patient»: Martin Krause.

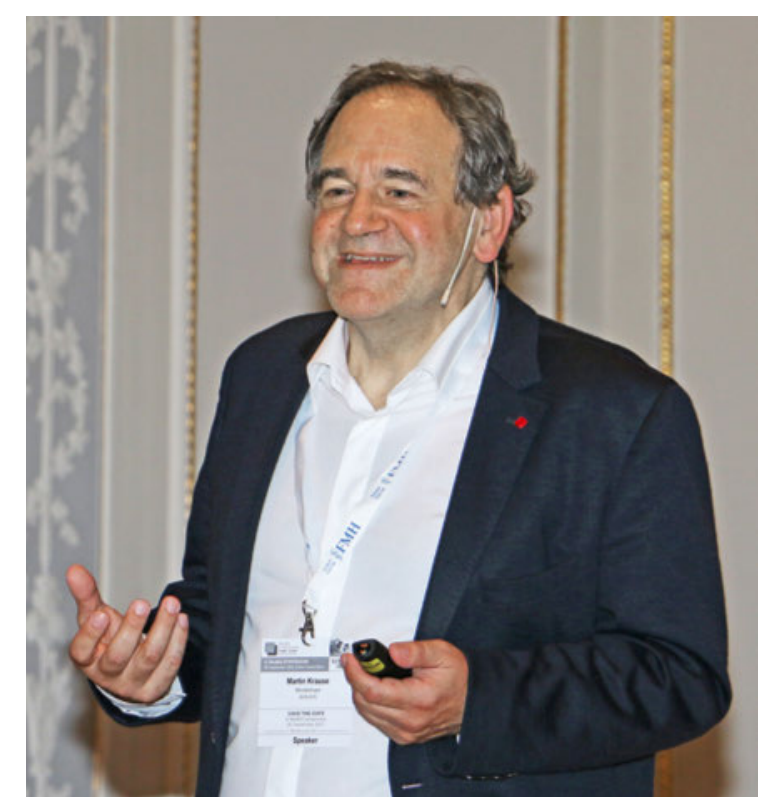

\section{ISFM-Award 2016: les lauréats}

Le troisième symposium MedEd a été I'occasion de révéler le nom des lauréats de I'ISFMAward 2016 pour un engagement exceptionnel dans la formation postgraduée, prix qui permet aux médecins-assistants de récompenser leurs anciens formateurs. Cette année, les formateurs suivants ont reçu une distinction:

Prof. Romaine Arlettaz, Zurich; Dr Stefan Arndt, Münsterlingen; Dr Esther Bächli, Uster; Dr Arménio Barata, Bienne; Dr Patrick Bodenmann, Lausanne; Dr Charles Dvorak, Vallorbe; Prof. Dominique Erni, Berne; Dr Bianka Freiwald, Baden; Prof. Emanuel Gautier, Fribourg; Prof. Andreas Günthert, Lucerne; Dr Samuel Henz, Saint-Gall/Rorschach; Dr Tobias Hübner, Münsterlingen; Dr Orpheus Kolokythas, Winterthour; Silvia Lambiel, Genève; Dr Adrian Marty, Zurich; Dr Stefan Mariacher-Gehler, Zollikerberg; Dr Friederike Meyer zu Bentrup, Viège; Dr Marco Negri, Frutigen; Dr habil. Sebastian Olbrich, Zurich; Prof. Nicolas Regamey, Lucerne; Dr lanina Scheer, Zurich; Dr Renato Scognamiglio, Fribourg; PD Dr Martin Siegemund, Bâle; Dr Nils Siegenthaler, Genève; Dr Daniel Studer, Bâle. 


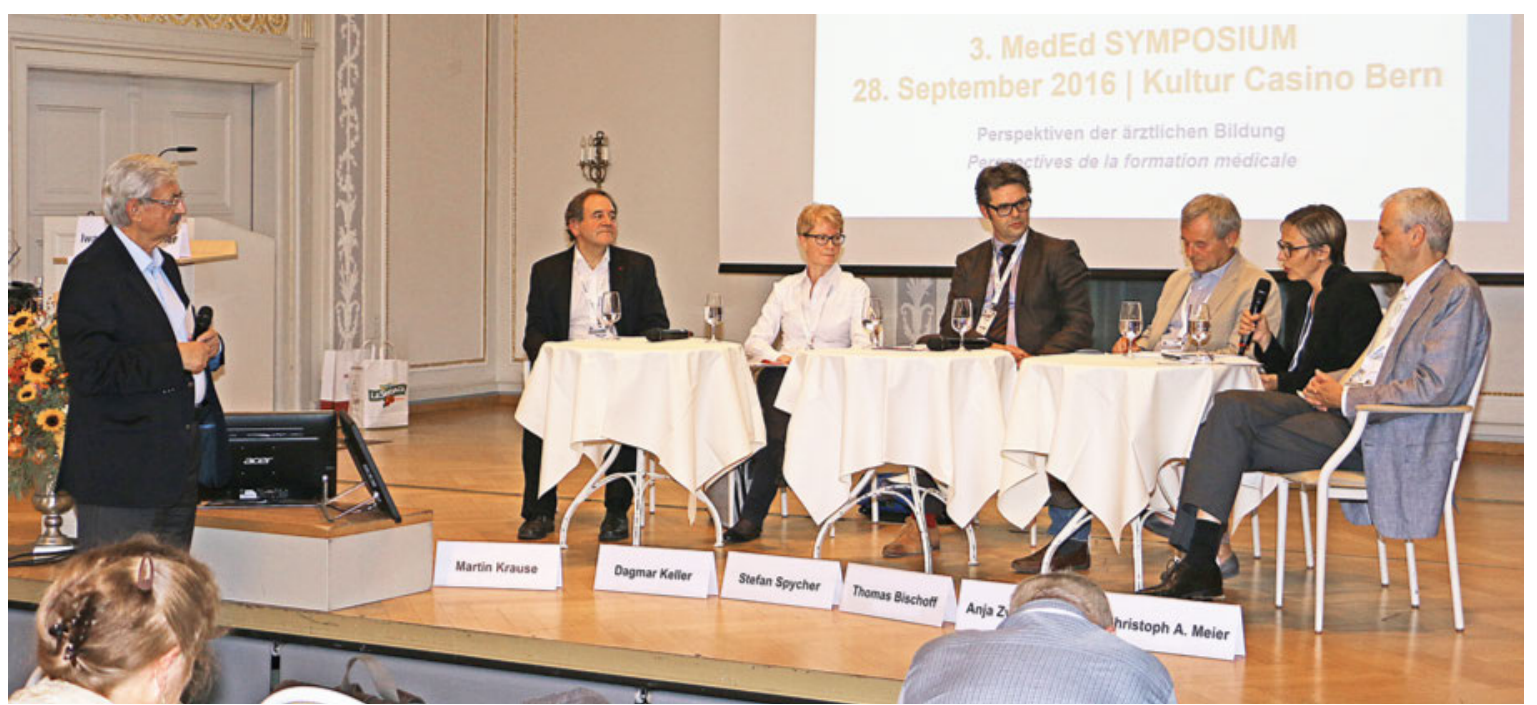

Une discussion engagée (de g. à d.): Iwan Rickenbacher (animateur), Martin Krause, Dagmar Keller, Stefan Spycher, Thomas Bischoff, Anja Zyska, Christoph A. Meier.

travail. La pression subtile exercée par les instances supérieures a contribué à améliorer la formation postgraduée au cours des dernières années.

La journée s'est conclue par une table ronde animée par Iwan Rickenbacher, l'occasion d'aborder des questions concrètes concernant la formation postgraduée. Les invités [3] ont débattu avec engagement des questions de la qualité, de la durée et du financement de la formation postgraduée, en s'intéressant également au changement des conditions de travail à l'hôpital et au cabinet, à l'évolution des attentes des patients et de la jeune génération de médecins, aux défis posés par le changement structurel ainsi qu'aux opportunités et aux risques du progrès technologique. Sur de nombreux points, les participants se sont montrés unanimes. Ils ont ainsi estimé qu'il serait souhaitable de réduire la durée de la formation postgraduée lorsque cela est possible. Ils ont également souligné l'importance du "Teaching" et le rôle modèle du formateur, sans oublier la pénurie de médecins de famille, qui exige des contre-mesures efficaces urgentes. A l'ère de la haute technologie, l'importance de l'empathie et de la proximité avec le patient a aussi été soulignée. «Nous ne devrions pas nous démarquer par une médecine high tech», a insisté Christoph A. Meier, «mais par une médecine raisonnable, axée sur le patient.»

Tous les participants au symposium s'accorderont certainement à dire que le débat sur la formation médicale n'est pas près de se tarir. Les thèmes à traiter ne manqueront pas non plus lors du $4^{\mathrm{e}}$ symposium MedEd qui se tiendra le 20 septembre 2017.

\section{Références}

1 Vous trouverez un résumé dans: Kesseli B. Se préparer efficacement aux défis de demain. Bull Méd Suisses. 2015;96(45):1638-41.

2 Les présentations des exposés et séminaires sont disponibles (en langue originale) à l'adresse http://www.fmh.ch/fr/formationisfm/themes/symposium-meded.html

3 La table ronde a réuni les personnes suivantes: Prof. Thomas Bischoff, vice-directeur de l'Ecole de formation postgraduée (EFPG) du CHUV, Lausanne; Prof. Dagmar Keller, directrice, Institut de médecine d'urgence, Hôpital universitaire de Zurich; Prof. Martin Krause, médecin-chef de la clinique médicale, Hôpital cantonal de Münsterlingen; Prof. Christoph A. Meier, directeur médical, Hôpital universitaire de Bâle; Dr Anja Zyska, vice-présidente de l'Association suisse des médecins-assistant(e)s et chef(fe)s de cliniques (ASMAC); Dr Stefan Spycher, vice-directeur, responsable de l'unité de direction Politique de la santé (OFSP).

\section{Crédits photo}

Photos Bruno Kesseli 


\section{SIWF ISFM}

\section{Améliorer l'apprentissage de l'auscultation cardiopulmonaire par les médecins-assistant-e-es}

L'auscultation est un geste central de l'examen clinique depuis l'invention du stéthoscope par Laennec en 1816. Deux cent ans plus tard, les avancées technologiques offrent de nouveaux moyens diagnostiques performants comme l'ultrasonographie aisément réalisable au lit du malade. Pourtant, l'auscultation a encore un rôle à jouer, d'autant plus qu'elle a elle-même bénéficié des progrès de la technologie. Ainsi, l'auscultation s'inscrit en complément des autres moyens diagnostiques disponibles.

L'auscultation cardiopulmonaire repose évidemment sur les compétences des médecins dans l'identification adéquate des sons. Or, plusieurs études montrent des lacunes dans ces compétences chez les médecins et chez les médecins-assistant-e-s en particulier.

Ce projet vise à améliorer les compétences des médecins-assistant-e-s dans l'auscultation cardiopulmonaire en mettant à profit les progrès technologiques qui ont marqué l'évolution du stéthoscope. Le stéthoscope électronique permet non seulement une transmission optimalisée des bruits cardiopulmonaires mais aussi l'accès simultané au phonospectrogramme sur un smartphone. Le médecin peut ainsi accéder à la combinaison des signaux acoustiques et visuels, avec une plusvalue en termes d'identification correcte des sons.

L'objectif du projet est de développer une application sur smartphone qui permette à des médecinsassistant-e-s d'accéder aux signaux acoustiques et visuels transmis en streaming à partir d'un stéthoscope électronique utilisé par un-e médecin superviseur lors d'un enseignement au lit d'une malade ou lors de la visite.
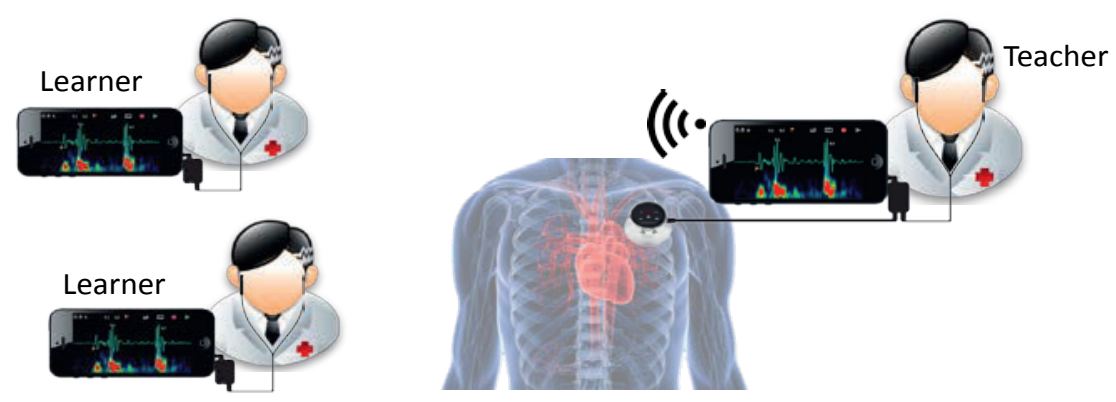

Ce projet est le fruit d'une collaboration entre le service de médecine interne du CHUV à Lausanne (Dr David Gachoud, Dr Matteo Monti et Prof. Gérard Waeber), I'unité pédagogique de la Faculté de Biologie et de Médecine de I'UNIL (Dr Raphaël Bonvin) et la société Smartcardia (Spin-Off de l'Ecole Polytechnique Fédérale de Lausanne ; Dr Srinivasan Murali et Dr Francisco Rincon). 


\section{SIWF ISFM}

\section{Introduction du Multisource Feedback (MSF) dans la formation médicale pré- et postgraduée}

Le cursus médical moderne exige, en plus d'une qualification constante des compétences techniques, la capacité de donner un feed-back structuré sur les compétences sociales et de communication des collaborateurs et des cadres assorti d'une vérification des progrès accomplis et de la formulation de nouveaux objectifs.

L'introduction du Multisource Feedback (MSF), établi depuis des décennies dans d'autres secteurs professionnels et parmi les cadres, permet de répondre à ce défi de manière adéquate et de nous faire évoluer vers la culture du travail de demain. Dans le système de santé anglais, le MSF est appliqué avec succès depuis de nombreuses années par le Royal College of Physicians.

La première phase du MSF s'adresse aux médecins-assistants. Chacun d'entre eux choisit 12 à 15 collaborateurs avec différentes fonctions (médecins-assistants, infirmières, médecins cadres) de son environnement professionnel, qui répondent anonymement à un questionnaire pour évaluer ses compétences sociales. Chaque médecin-assistant procède aussi à une auto-évaluation. La synthèse de cette évaluation et de celle de ses collègues est discutée avec un tuteur (un médecin cadre spécialement formé au feed-back) afin d'identifier les potentiels individuels et fixer de nouveaux objectifs. Ce feed-back est régulier.

Le MSF doit être efficace et donner des résultats clairs et exploitables. Le questionnaire se remplit en ligne de manière anonyme et le résultat est évalué automatiquement. Pour la mise en œuvre de cette partie, nous collaborons avec une société spécialisée qui a beaucoup d'expérience dans ce domaine.

Pendant la deuxième phase, le MSF se concentre sur les cadres. Le processus demande encore quelques adaptations. Des coachs externes viennent s'entretenir avec les cadres sur les résultats du feed-back. Cela permet une meilleure acceptation et de meilleurs résultats.

Il est prévu, dans une troisième phase, d'appliquer le MSF à tout l'Hôpital cantonal de Winterthur (KSW) puis à d'autres hôpitaux. 


\title{
SIWF \\ ISFM
}

\section{Développement et implémentation d'une plateforme générique électronique pour les Work-place Based Assessments - eWPBA}

\author{
Dr Matthias Hepprich ${ }^{1}$, Dr Gianmarco M. Balestra ${ }^{2,3}$
}

Les évaluations en milieu de travail (WPBA) comme les Mini-CEX et les DOPS ont été introduites en Suisse pour améliorer la qualité de la formation médicale postgraduée. Elles servent de base à la supervision et au feed-back des étudiants et des médecins-assistants. Leur objectif est d'optimiser la manière d'acquérir les compétences médicales. Pour cela, le feed-back des cliniciens est décisif mais de nombreux obstacles viennent compliquer une application efficace des WPBA.

Les principales critiques dirigées contre les WPBA sont le temps à investir, les ressources humaines requises, la nécessité de remplir des formulaires papier, le manque de connaissances des outils ou des critères d'évaluation, et le manque d'explications sur la manière de donner un bon feed-back.

Alors que le temps et le personnel sont des composantes inhérentes au format de l'exercice, les autres aspects sont susceptibles d'être modifiés. Nous avons développé une application facile à utiliser qui permet une évaluation structurée. Avec la plateforme électronique générique prévue, il est possible d'améliorer la qualité et le temps d'exécution des WPBA. De plus, les données des WPBA sont disponibles à long terme et de manière plus détaillée tout au long de la phase d'apprentissage et pour l'analyse générale. La version alpha peut être utilisée sur les iPhones $®$ et elle devrait être prochainement compatible avec les autres appareils portables. Nous attendons une meilleure applicabilité et acceptabilité dans le quotidien clinique grâce à la simplification du processus d'évaluation, à une baisse de la charge administrative et à une incitation à un feed-back différencié. Après la phase de projet, l'application sera utilisée et évaluée à l'Hôpital universitaire bâlois. Le design et l'architecture logicielle sur laquelle il repose ont été conçus de sorte à pouvoir être largement utilisés dans le domaine de la formation prégraduée et postgraduée d'autres hôpitaux suisses. Les interfaces avec d'autres logiciels (p. ex. le logbook de la FMH) ont été prises en compte et offrent des possibilités d'intégration.

\footnotetext{
${ }^{1}$ Clinique d'endocrinologie, diabétologie et métabolisme, Hôpital universitaire bâlois, Petersgraben 4, 4031 Bâle

${ }^{2}$ Soins intensifs, Hôpital universitaire bâlois, Petersgraben 4, 4031 Bâle

${ }^{3}$ Décanat de la Faculté de médecine, Hôpital universitaire bâlois, Klingelbergstrasse 61, 4056 Bâle
} 